\title{
EXAME OBSTÉTRICO REALIZADO PELA ENFERMEIRA: DA TEORIA À PRÁTICA
}

Maryanne Machado Ataide ${ }^{1}$ Amuzza Aylla Pereira dos Santos², Jovânia Marques de Oliveira e Silva², Maria Elisângela Torres de Lima Sanches ${ }^{4}$

Objetivo: analisar o conhecimento e a prática da enfermeira acerca do exame físico obstétrico. Metodologia: pesquisa descritiva, quantitativa, realizada com 24 enfermeiras que atuam na assistência obstétrica em maternidades públicas da cidade de Maceió/ AL, no período de junho a agosto de 2014. Foi aplicado um questionário e, após essa etapa, realizada a análise descritiva dos dados. Resultados: o exame das mamas e a palpação obstétrica são as técnicas mais negligenciadas pela enfermeira durante a realização do exame obstétrico. As únicas técnicas realizadas unanimemente por elas são a medição da altura de Fundo de útero e o toque vaginal. Conclusão: o exame obstétrico realizado pela enfermeira ainda é subutilizado nas parturientes. As enfermeiras demonstram conhecimento sobre o assunto, porém a prática realizada não condiz com a teoria informada.

Descritores: Enfermagem; Obstetrícia; Assistência; Gestante.

\section{EXAMINATION EXAM BY OBSTETRIC NURSE: FROM THEORY TO PRACTICE}

Objective: analyze the knowledge and practice nurse about obstetrical physical examination. Methodology: descriptive, quantitative research among 24 nurses working in maternity care in public hospitals of the city of Maceió/AL from June to August 2014. It was used a questionnaire containing demographic data, working time and knowledge exam. After this step was carried out a descriptive analysis of data. Results: The breast examination and obstetric palpation are the most neglected by nurses techniques while performing the obstetrical examination. The only techniques unanimously carried by them are Asao measuring the height of the uterus Fund and the vaginal ring. Conclusion: Obstetric examination performed by the nurse is still underused in pregnant women. Nurses demonstrate knowledge on the subject, but the practice performed dismissive informed theory.

Descriptors: Nursing; obstetrics; assistance; pregnant.

\section{EXPLORACIÓN OBSTETRICA REALIZADO POR LA ENFERMERA DE LATEORÍAA LA PRÁCTICA}

Objetivo: analizar el conocimiento y la práctica enfermera acerca de la exploración física obstétrica. Metodología: Estudio descriptivo, la investigación cuantitativa entre 24 enfermeras que trabajan en la atención de maternidad en los hospitales públicos de la ciudad de Maceió/AL de junio a agosto de 2014. Se utilizó un cuestionario que contiene datos demográficos, tiempo de trabajo y el conocimiento examen. Después de este paso se llevó a cabo un análisis descriptivo de dados. Resultados: El examen de las mamas y la palpación obstétrica son los más desatendidos por técnicas enfermeras mientras se realiza el examen de gravidez. Las únicas técnicas realizadas por unanimidad por ellos son Asao medir la altura del fondo del útero y el anillo vaginal. Conclusión: Examen obstétrico realizado por la enfermera todavía no se usa mucho en las mujeres embarazadas. Las enfermeras demuestran conocimiento sobre el tema, pero la práctica la teoría realizaron informado desdeñoso.

Descriptores: Enfermería; Obstetricia; Asistencia; Mujer embarazada.

${ }^{1}$ Acadêmica de Enfermagem da Escola de Enfermagem e Farmácia da Universidade Federal de Alagoas (ESENFAR/UFAL). E-mail: maryanne-machado@hotmail.com. ${ }^{2}$ Doutoranda do Programa de Pós-Graduação em Ciências da Saúde da Universidade Federal de Alagoas. Docente da ESENFAR/UFAL.

${ }^{3}$ Enfermeira. Doutora em Enfermagem pela Universidade Federal da Bahia. Docente da ESENFAR/UFAL.

${ }^{4}$ Enfermeira. Especialista em Enfermagem obstétrica. Docente da ESENFAR/UFAL. 


\section{INTRODUÇÃO}

A atenção à saúde materna e infantil requer a oferta de serviços de qualidade que garantam acesso facilitado por meio do cumprimento dos protocolos estabelecidos para uma assistência baseada nas melhores evidências científicas que proporcione intervenções oportunas para garantir a saúde do binômio. A cobertura da atenção com qualidade ao parto das mulheres faz com que os profissionais de saúde utilizem técnicas que consigam identificar precocemente algumas das complicações que podem ocasionar o aumento das taxas de mortalidade materna ${ }^{(1,2)}$.

Nessa perspectiva, um dos profissionais que presta assistência durante o ciclo gravídico-puerperal é a enfermeira, que precisa estar qualificada, para detecção precoce dos agravos relacionados ao parto e nascimento, bem como para assistir adequadamente quando forem necessários os cuidados imediatos para diminuição da taxa de morbimortalidade materno-infantil. O acompanhamento inadequado, sem avaliações regulares, é um dos motivos do alto índice da taxa de mortalidade materno-infantil, demonstrando que as diversas intervenções realizadas não garantem a qualidade da assistência obstétrica prestada(3).

Desse modo, a qualidade dos serviços obstétricos prestados pela enfermeira assume um importante papel para o alcance de melhorias da assistência prestada no trabalho de parto. No entanto, para a qualidade do exame obstétrico, há necessidade de conhecimento das técnicas realizadas, bem como do processo utilizado para organização do serviço e necessidades da parturiente ${ }^{(4,5)}$.

Para que a assistência qualificada seja prestada durante o ciclo, a enfermeira dispõe do Processo de Enfermagem (PE) que é o modelo metodológico aplicado por meio de conhecimentos técnico-científicos na prática assistencial. Esse processo é desenvolvido em várias etapas: inicialmente, é feita a anamnese, em que são registradas a história pregressa das gestantes, incluindo antecedentes obstétricos, data da última menstruação (DUM); informações sobre a movimentação fetal; dados referentes à evolução da gestação atual; e exame físico obstétrico das gestantes ${ }^{(6,7)}$.

As demais etapas do processo são: Diagnósticos de Enfermagem - elaborado mediante os fatos elencados na primeira etapa (anamnese e exame físico); o planejamento de enfermagem e a implementação da assistência de Enfermagem, por meio da prescrição de Enfermagem, a avaliação da assistência de Enfermagem que consiste em acompanhar e registrar as respostas da gestante aos cuidados, avaliando os resultados das prescrições de enfermagem implementadas ${ }^{(6,7)}$

O exame físico obstétrico inicia-se com a inspeção obstétrica, que será realizada na sequência cefalocaudal. Após a inspeção, deve haver a mensuração da altura uterina para averiguação do crescimento do útero para posterior comparação com a idade gestacional da gestante ${ }^{(5)}$. A palpação obstétrica é então realizada a fim de se reconhecer a situação, posição, apresentação e insinuação fetal. Porém, pela palpação manual, apenas é possivel avaliar a frequência e a duração das contrações. Caso haja desvios da contratilidade uterina normal, é necessária a intervenção a fim de corrigi$\mathrm{la}^{(8)}$.

Em seguida, o toque vaginal é realizado, sendo fundamental para avaliação do progresso do trabalho de parto. Permite definir a dilatação do colo uterino, a posição, comprimento, consistência e tamanho do colo, apresentação do feto, atitude e posição fetal, presença de bossa e grau de cavalgamento ósseo do crânio fetal, relação entre a apresentação e o colo uterino, características da bacia óssea materna, presença de membranas e sua reação às contrações uterinas. Porém, o toque vaginal deve ser realizado o menor número de vezes possiveis, visto que o manuseio excessivo do canal de parto está relacionado com a morbidade infecciosa e a ruptura das membranas ovulares ${ }^{(9)}$.

Após a realização do exame obstétrico, devem ser registrados formalmente os dados coletados durante a consulta de enfermagem, os diagnósticos de enfermagem identificados a partir da coleta de dados, bem como as ações realizadas e os resultados alcançados ${ }^{(9)}$. Os registros de enfermagem possibilitam uma adequada comunicação entre a equipe de enfermagem e os demais profissionais envolvidos no cuidar, pois servem de base para a elaboração do plano assistencial. Além disso, fornecem subsídios para a avaliação da assistência prestada, acompanhamento da evolução obstétrica, favorecem a auditoria de enfermagem, colaboram com o ensino e a pesquisa, e tornam-se importantes instrumentos, que devem ser consultados, em situações que envolvem aspectos legais e/ou éticos ${ }^{(10)}$.

Após a busca nos bancos de dados eletrônicos sobre o exame obstétrico realizado pelos enfermeiros em parturientes e a frequência de seus registros, percebeu-se uma deficiência de estudos sobre essa temática. Mediante o exposto, o estudo teve como objetivo analisar o conhecimento e a prática da enfermeira acerca do exame físico obstétrico.

\section{METODOLOGIA}

Estudo descritivo, exploratório, com abordagem quantitativa, realizado em duas instituições públicas, e uma filantrópica da cidade de Maceió-AL, no período de junho a agosto de 2014. A população foi constituída por 24 enfermeiras que totalizam o quantitativo de profissionais responsáveis em prestar assistência na sala de pré-parto e parto das instituições pesquisadas. Para a coleta de dados, foi elaborado um questionário, com dados sociodemográficos e dados referentes ao conhecimento e a prática da enfermeira 
acerca do exame físico obstétrico.

Os dados foram coletados após aprovação do projeto pelo Comitê de Ética sob o no 30253114.0.0000.5013 e a assinatura do Termo de Consentimento Livre Esclarecido pelo entrevistado. As 24 enfermeiras, todas do sexo feminino, prestam atendimento às parturientes no pré-parto e na triagem das maternidades das três instituições de saúde, identificadas na pesquisa como Instituições de Saúde A, B e C, situadas na cidade de Maceió, no estado de Alagoas. Sendo $\mathrm{A}$ e $\mathrm{B}$ públicas e $\mathrm{C}$ filantrópica. Entre as enfermeiras que participaram da pesquisa, cinco estão atuando na instituição de saúde "A", sendo todas enfermeiras generalistas (EG); seis na instituição de saúde "B", e 13 na instituição de saúde " $C$ ". Todas as enfermeiras entrevistadas da Instituição de Saúde B e C são enfermeiras obstétricas (EO)(19).

Para a organização, tabulação e análise dos dados descritivos utilizou-se o programa Microsoft Excel 2010. A análise descritiva ocorreu a partir de frequência absoluta (n) e os resultados foram apresentados de forma descritiva e por meio de tabelas.

\section{RESULTADOS}

A maioria das 24 enfermeiras foi graduada em instituições públicas $(83,3 \%)$ e estão formadas entre cinco e nove anos (37,5\%), ganham entre dois e quatro salários mínimos (53\%), possuem até um vínculo empregatício $(66,7 \%)$, e atuam apenas em gestação de risco habitual (50\%). A maioria realizou especialização na área $(79,2 \%)$ e faz atualizações periódicas nesse tema.

Quando questionadas acerca de saber realizar o exame obstétrico completo na parturiente, as 19 EO afirmaram saber realizá-lo; duas das cinco EG responderam que não sabem realizá-lo e as demais, que sabem realizar, porém de forma incompleta. Em relação ao modo de realizar o exame obstétrico completo na parturiente, as técnicas que as enfermeiras afirmaram realizar durante esse exame estão expostas na Tabela 1.

Tabela 1 -Técnicas realizadas pelas EO e EG nas parturientes durante o exame obstétrico. Maceió, AL, 2015.

\begin{tabular}{|l|r|r|r|r|}
\hline \multirow{2}{*}{ Técnicas realizadas } & & EO & & EG \\
\cline { 2 - 5 } & Sim & Não & Sim & Não \\
\cline { 2 - 5 } & N & N & N & N \\
\hline Exame das mamas & 3 & 16 & 4 & 1 \\
\hline Palpação obstétrica & 12 & 7 & 0 & 0 \\
Medição da Altura de Fundo & 19 & 0 & 1 & 4 \\
\hline
\end{tabular}
de útero (AFU)
Ausculta dos Batimentos Cardíacos Fetais (BCF)

Avaliação da Dinâmica uterina (DU)

Exame especular

Toque Vaginal

Na Tabela 2, estão distribuídos os números de EO e EG que avaliam e interpretam o resultado das técnicas mais citadas durante o exame obstétrico, de acordo com o parâmetro de normalidade preconizado pelo Ministério da Saúde(9), bem como, as que realizam essas técnicas na frequência correta.

Tabela 2 - EO e EG que realizam as técnicas nas parturientes com frequência e interpretação conforme preconizado pelo Ministério da Saúde. Maceió, AL, 2015.

\begin{tabular}{|l|l|l|l|l|l|l|}
\hline $\begin{array}{l}\text { Dado } \\
\text { Técnica }\end{array}$ & \multicolumn{2}{|l|}{$\begin{array}{l}\text { Número de } \\
\text { profissionais }\end{array}$} & \multicolumn{2}{l|}{ Interpretam } & \multicolumn{2}{l|}{$\begin{array}{l}\text { Frequência } \\
\text { correta }\end{array}$} \\
\hline & EO & EG & EO & EG & EO & EG \\
\cline { 2 - 7 } & 19 & 1 & 19 & 1 & $*$ & $*$ \\
\hline AFU & 18 & 1 & 18 & 0 & 18 & 0 \\
\hline BCFs & 18 & 3 & 18 & 3 & 13 & 0 \\
\hline DU & 19 & 0 & 19 & - & 17 & - \\
\hline $\begin{array}{l}\text { Toque } \\
\text { vaginal }\end{array}$ & & & & & & N \\
\hline
\end{tabular}

* Não existe frequência preconizada para essa variável.

Com relação às dificuldades enfrentadas pelas EO para realizar o exame obstétrico completo na parturiente, seis enfermeiras negaram ter qualquer dificuldade; em contrapartida, 13 citaram ter pelo menos uma dificuldade. Das EG, cinco citaram pelo menos uma dificuldade em realizar o exame obstétrico completo na parturiente.

Com relação aos instrumentos de registros que as instituições de saúde dispõem para os enfermeiras, a instituição "A" disponibiliza às enfermeiras apenas a folha de evolução para os registros dos dados. Já as instituições de saúde "B" e "C" disponibilizam, além da folha de evolução do prontuário, a ficha de triagem - em que ficam registrados os dados da anamnese e do exame obstétrico realizados durante a admissão da parturiente e o partograma.

Acerca da regularidade do registro, duas EG e 15 EO afirmaram registrar regularmente todos os dados coletados durante o exame obstétrico, apresentando diferentes motivos (Tabela 3). 
Tabela 3 - Distribuição dos motivos para registrar os dados coletados no exame obstétrico. Maceió, AL, 2015.

\begin{tabular}{|c|c|c|}
\hline \multirow[t]{2}{*}{ Motivo } & EO & EG \\
\hline & $N=15$ & $N=2$ \\
\hline Segurança jurídica e respaldo profissional & 6 & 1 \\
\hline $\begin{array}{l}\text { Para que outros profissionais possam ter } \\
\text { acesso }\end{array}$ & 9 & 1 \\
\hline $\begin{array}{l}\text { Acompanhar a evolução do trabalho de } \\
\text { parto }\end{array}$ & 3 & - \\
\hline Não esquecer os dados avaliados & 2 & - \\
\hline $\begin{array}{l}\text { Para que a cliente tenha acesso aos } \\
\text { registros }\end{array}$ & 1 & - \\
\hline
\end{tabular}

\section{DISCUSSÃO}

Destaca-se neste estudo que as enfermeiras possuem apenas um vínculo empregatício, dado semelhante a estudo(11) realizado em Londrina/PN, onde $100 \%$ dos profissionais de enfermagem na assistência obstétrica possuem apenas um emprego. Ainda, enfermeiras graduadas em instituições públicas de ensino superior com formação relativamente recente - menos de nove anos - o que sugere que estejam atualizadas com relação às necessidades atuais da assistência obstetrícia, com enfoque na atenção humanizada e de qualidade.

Com relação à complexidade da assistência, as entrevistadas trabalham apenas com assistência à gestação de risco habitual, em que as necessidades das gestantes que não apresentam agravos durante a gravidez são resolvidas com procedimentos simples no nível primário de assistência. De acordo com o Ministério da Saúde ${ }^{(12)}$, para o atendimento às gestantes de risco habitual, a instituição de saúde deve contar com uma equipe detentora de conhecimentos, que oferte uma assistência de qualidade. Essa clientela necessita de cuidados que vão desde o mínimo, como para aquelas que apresentam poucos riscos de sofrerem danos, até os cuidados máximos, pela alta probabilidade de evolução desfavorável, para a mãe e o feto.

Sendo o exame obstétrico de suma importância para a detecção precoce de possiveis complicações no momento do parto e para a tomada de condutas necessárias, as EO devem utilizar os seus conhecimentos teórico-práticos adquiridos para realizar o exame obstétrico, como preconizado pela literatura ${ }^{(11)}$.

Os dados mostram que a realização do exame de mamas pelas $E \mathrm{O}^{(6)}$ merece destaque, visto que esse é um exame obstétrico importante para detectar as condições da mama, mamilos, presença do colostro, presença de fissuras, dentre outros. Ainda, o contato cutâneo direto e precoce entre mãe e filho, e o apoio ao início da amamentação na primeira hora após o parto são práticas que devem ser estimuladas e, para isso, o exame das mamas deve ser realizado em todas as gestantes/parturientes atendidas ${ }^{(13)}$.

A palpação obstétrica, ou Manobra de Leopold, merece mais atenção por parte dos profissionais, visto que $12 \mathrm{EO}$ realizam essa manobra, porém nenhuma EG a faz. É necessário atentar que, a partir dessa manobra, há a identificação da situação e da apresentação fetal e, ainda, há a estimativa da quantidade de líquido amniótico, importante para a identificação de anormalidades e situações de risco para o feto e para a tomada de decisões durante o trabalho de parto (13).

Todas as EO afirmaram sempre medir a AFU. Além disso, 17 delas reconhecem os parâmetros de normalidade como preconizados pelo Manual do Pré Natal e Puerpério do Ministério da Saúde ${ }^{(12)}$. Esse refere a partir da 20a semana, existe relação direta entre as semanas da gestação e a medida da altura uterina. Porém, esse parâmetro torna-se menos fiel a partir da 30a semana de idade gestacional. Além disso, se o feto estiver em situação transversa, a medida da altura uterina irá diminuir, podendo falsear sua relação com a idade gestacional.

O fato de 18 EO afirmarem que sempre auscultam e avaliam os BCFs, durante a assistência à parturiente, é muito positivo, pois é a partir da sua ausculta que é realizada a constatação da presença, do ritmo, da frequência, e de qualquer anormalidade dos BCFs, havendo, assim, a avaliação da vitalidade fetal. Porém, deve ser destacado que apenas uma EG afirmou realizar essa técnica, sendo transferida a responsabilidade dessa avaliação para o profissional médico.

Com relação à avaliação da DU, 18 EO enfermeiras obstétricas e três EG a realizam. Porém, apenas 13 dessas EO a avaliam na frequência preconizada pela literatura. $\mathrm{Na}$ fase latente do trabalho de parto e no início da fase ativa, o controle da DU pode ser feito a cada 60 minutos, reduzindo esse intervalo para 30 minutos, conforme progride a fase ativa do trabalho de parto ${ }^{(14)}$. Deve-se destacar, também, que nenhuma das EG avalia a DU nessa frequência.

A grande maioria das EO (17) realiza o toque vaginal na frequência preconizada pelo Ministério da Saúde ${ }^{(12)}$, ou seja, a cada duas horas. Nos casos suspeitos ou confirmados de amniorrexe prematura, no entanto, as mulheres não devem ser submetidas ao exame de toque vaginal, porque isso aumenta o risco de infecções amnióticas, perinatais e puerperais ${ }^{(15)}$.

Nenhuma EG realiza o toque vaginal sob a justificativa da falta de especialização e respaldo profissional para realização dessa técnica. Porém, legalmente, a enfermeira poderá realizar, como integrante da equipe de saúde, o toque vaginal(14). Uma EO citou o exame especular como parte da assistência prestada à parturiente e esse fato merece atenção. O Ministério da Saúde ${ }^{(12)}$ preconiza que, quando existe suspeita de amniorrexe prematura ou sangramento genital, o 
exame especular deve ser rotina, precedendo a decisão pela realização do toque. Porém, este estudo demonstrou que 18 EO entrevistadas priorizam a realização do toque vaginal ao especular.

Este estudo revela que as técnicas mais citadas pelas enfermeiras estão em consonância com as atividades executadas com maior frequência durante o trabalho de parto. Contudo, todas as técnicas que compõem o exame físico obstétrico devem ser realizadas em todas as parturientes, para que os trabalhos de parto sejam bem avaliados e sejam tomadas as condutas certas no momento adequado, sem intervenções desnecessárias ${ }^{(9-11)}$.

Esses achados permitiram verificar que, apesar da especialização, muitas habilidades básicas que compõem o exame obstétrico deixam de ser realizadas por algumas EO, as quais deveriam prezar pela forma completa do exame obstétrico, pois essas condutas podem ajudar na detecção precoce de agravos que podem complicar o trabalho de parto, levando ao óbito materno e fetal(15). Por outro lado, as EG apresentam dificuldades para realizar o exame obstétrico, demonstrando que a falta do curso de especialização em obstetrícia pode prejudicar a assistência prestada nesses setores.

O não registro das ações de enfermagem é um fato preocupante, visto que os registros de enfermagem consistem em uma forma de comunicação escrita de informações pertinentes ao cliente e aos cuidados recebidos pela equipe de enfermagem. São elementos essenciais na documentação e, quando redigidos adequadamente, possibilitam a efetiva comunicação entre a equipe de enfermagem e os demais profissionais. Também possibilitam a continuidade do cuidado, uma vez que oferecem, a todos os membros da equipe de saúde, informações sobre os problemas de saúde identificados, ações e resultados dos cuidados prestados ${ }^{(10)}$.

O estudo apresentou limitações referentes à dificuldade das enfermeiras em responder algumas questões acerca das práticas utilizadas para o exame obstétrico.

\section{CONCLUSÃO}

O exame obstétrico realizado pelas enfermeiras ainda é subutilizado nas parturientes. EO demonstram conhecimento sobre o assunto, porém a prática realizada não parece condizer com o conhecimento informado. Há um quantitativo de EG nos serviços de atendimento obstétrico, confluindo para a não efetividade dos exames obstétricos necessários.

Os serviços de saúde devem estimular e oferecer aos seus profissionais cursos de aprimoramento na atenção obstétrica, a fim de que haja melhoria da qualidade do atendimento às parturientes e diminuição das altas taxas de morbimortalidade materna e fetal

\section{REFERÊNCIAS}

1. Leal MC, Pereira APE Domingues RMSM, Theme-Filha MM, Dias MAB, Nakamura-Pereira M, Bastos MH, Gama SGN. Intervenções obstétricas durante o trabalho de parto e parto em mulheres brasileiras de risco habitual. Cad Saúde Pública. 2014; 30 (Supl.): S17-32.

2. Leal MC, Theme-Filha MM, Moura EC, Cecatti JG, Santos LMP. Atenção ao pré-natal e parto em mulheres usuárias do sistema público de saúde residentes na Amazônia Legal e no Nordeste, Brasil 2010. Rev. Bras. Saude Mater. Infant. 2015 Mar [cited 2016 Aug 15] : 15( 1 ): 91-104. Available from: http://www.scielo.br/scielo.php?script=sci_arttext\&pid=S1519382920150001000918lng=en. http://dx.doi.org/10.1590/S151938292015000100008

3. Cunha MA, Namede MV, Dotto LMG, Namede FV. Assistência prénatal: competências essenciais desempenhadas pos. Rev. Esc Ana Nery. 2009:13(1):145-53

4. Vasconcelos KL, Martins CA, Mattos DV, Tyrrell MAR, Bezerra ALQ, Porto J. Partograma: instrumento para segurança na assistência obstétrica. J. Nurs UFPE on line. 2013; [cited 2016 Aug 15];7 (2): 619-24. Available from: http://www.revista.ufpe.br/revistaenfermage. m/index.php/revista/article/ view/4739/pdf_2997 DOI: 10.5205/reuol

5. Bittencourt SDA, Reis LGC, Ramos MM, Rattner D, Rodrigues PL, Neves DCO et al . Estrutura das maternidades: aspectos relevantes para a qualidade da atenção ao parto enascimento. Cad. Saúde Pública. 2014 [cited 2016 Aug 15] ; 30( Suppl 1): S208-S219. Available from: http://www.scielo. br/scielo.php?script=sci_arttext\&pid=S0102-311X2014001300025\&lng=en. http://dx.doi.org/10.1590/0102-311X00176913.

6. Narchi NZ. Atenção pré-natal na Zona Leste da cidade de São Paulo Brasil. Rev. Esc. Enferm. USP. 2010; 44(2): 266-73.

7. Viellas EF, Domingues RMSM, Dias MAB, Gama SGN, Theme-Filha MM,
Costa JV, Bastos MH, Leal MC. Assistência pré-natal no Brasil. Cad Saúde Pública. 2014; 30 (Supl.): S85-S100.

8. Santos RB, Ramos KS. Sistematização da assistência de enfermagem em Centro Obstétrico. Rev. Bras. Enferm. 2012; 65 (1):13 - 8.

9. Busanello J. Kerberll NPC, Sassi Ram, Mano OS, Susin LRO, Gonçalves BG. Atenção humanizada ao parto de adolescentes: análise das práticas desenvolvidas em um Centro Obstétrico. Rev. Bras. Enferm. 2011:64 (5): 824-32.

10. Barros LA, Verissimo RCSS. Uso do partograma em maternidades escola de alagoas. Rev. Rene. 2011;12 (3): 555-60.

11. Barral LNM, Ramos LH, Vieira MA, Dias OV, Souza LPS. Análise dos registros de enfermagem em prontuários de pacientes em um hospital de ensino. Rev. Min. Enferm. 2012; 16(2): 188-93.

12.Brasil. Ministério da Saúde. Caderno de atenção ao Pré-natal e puerpério - atenção qualificada e humanizada. Manual Técnico. Brasilia: Editora do Ministério da Saúde, 2012.

13. Esser MAMS, Mamede FV, Mamede MV. Perfil dos profissionais de enfermagem que atuam em maternidades em Londrina, PR. Rev. Eletr. Enf 2012; 14(1): 133-41.

14. Pieszak GM, Terra MG, Neves ET, Pimenta LF, Padoin SMM, Ressel LB. Percepção dos profissionais de enfermagem acerca do cuidar em centro obstétrico. Rev Rene. 2013:14(3): 568-78.

15.Cagnin ERG, Mamede MV, Mamede FV. Atenção qualificada ao trabalho de parto: um estudo descritivo. J. Nurs UFPE on line. 2014;10 [cited 2015 Jul 25]; (8): 3266- 74. Available from: http://www.revista.ufpe.br/ revistaenfermage. $\mathrm{m} /$ index.php/revista/article/view/4739/pdf_ 2997 DOI: $10.5205 /$ reuol 